\title{
Smart signal processing for an evolving electric grid
}

\author{
Leandro Rodrigues Manso Silva ${ }^{1 *}$, Calos Augusto Duque ${ }^{1}$ and Paulo F Ribeiro ${ }^{2}$
}

\begin{abstract}
Electric grids are interconnected complex systems consisting of generation, transmission, distribution, and active loads, recently called prosumers as they produce and consume electric energy. Additionally, these encompass a vast array of equipment such as machines, power transformers, capacitor banks, power electronic devices, motors, etc. that are continuously evolving in their demand characteristics. Given these conditions, signal processing is becoming an essential assessment tool to enable the engineer and researcher to understand, plan, design, and operate the complex and smart electronic grid of the future. This paper focuses on recent developments associated with signal processing applied to power system analysis in terms of characterization and diagnostics. The following techniques are reviewed and their characteristics and applications discussed: active power system monitoring, sparse representation of power system signal, real-time resampling, and time-frequency (i.e., wavelets) applied to power fluctuations.
\end{abstract}

Keywords: Smart grid; Power system signal processing; Smart monitoring; Active monitoring

\section{Introduction}

The increasing complexity of the electric grid requires intensive and comprehensive signal monitoring followed by the necessary signal processing (SP) for characterizing, identifying, diagnosing, protecting, and also for better unfolding the nature of certain phenomena and events. Signal processing can also be used for predicting and anticipating system behaviors; thus, SP for electrical engineering is a vital tool for separating, decomposing, and revealing different aspects and dimensions of the complex physical operation of electrical systems, in which different phenomena are usually intricately and intrinsically aggregated and not trivially resolved.

As the electric grid becomes more multifaceted, modeling and simulation become less capable of capturing the influence of the multitude of independent components within the network. Since SP deals with the actual system (no modeling abstraction or reduction, though it may be used in connection with simulations), it encompasses all without clashing and as such mediates its unity through a great diversity of aspects. Consequently,

\footnotetext{
* Correspondence: leandro.manso@ufjf.edu.br

${ }^{1}$ Federal University of Juiz de Fora, Juiz de Fora, MG, Brazil

Full list of author information is available at the end of the article
}

SP can detect and reveal the true nature of the electric grid behavior.

The aim of this paper is to present some useful power system signal processing applications in the context of smart grids. At the same time, it presents well-established SP tools applied to a power system and it introduces new concepts that would be helpful in the smart grids. "Section 2 " presents the concept of active power system monitoring and processing. In this concept, an external and controlled signal is injected into the electric network and the behavior of the system due to this external stimulus is acquired and information is extracted using signal processing. Although the idea can be applied for extracting a large set of information, "Section 2" focuses on network impedance estimation, which is a practical example of active monitoring and signal processing, and it is an important parameter to be estimated in the smart grid context.

"Section 3" deals with the sparse representation of power system signal. The sparse representation, over redundant dictionary, has emerged as a potential method for detection, estimation, and compression of power system signal. Although the section focuses on signal compressing application, the authors wish to draw attention to the potential of the method for other applications, such as detection and classification. A special case comparing the compression 
rate between the well-established wavelet transform and the sparse representation is presented in this section.

"Section 4" covers the topic of real-time resampling technique, specifically the one when the fundamental frequency varies with time. In this case, the concept of arbitrary sampling rate interpolation (ASRI) is used. This concept requires a frequency estimator and uses an adaptive interpolator filter in order to synchronize the signal in real time. The adaptive filter can run using batch or sample by sample processing. After real-time synchronization, the harmonic components can be estimated using the regular fast Fourier transform (FFT) and the harmonic and subharmonic groups and subgroups can be computed according to the standard. An example showing that ASRI reduces drastically the estimation error is also discussed.

"Section 5" explores the application of time-frequency analysis (wavelet transform-WT) in generation and load profiles. Through the analysis of relevant signals, the variations in the signals of different frequency ranges can be characterized. In this section, the power fluctuations in a small $10-\mathrm{kV}$ distribution network (acting as a microgrid) are analyzed using WT and the results show that the tool is useful both for the characterization of possible electricity storage devices and the determination of the required balancing capacities.

Finally, "Section 6" presents the conclusions of this paper.

\section{Passive versus active power system monitoring and processing}

Electrical parameter estimation like fundamental frequency, phasor values, and harmonic components, among others, is a significant piece of information used to diagnose the state of the electrical system regarding possible solutions to restore the system health. Figure 1 illustrates a comprehensive system-wide signal processing analysis. This figure shows that if the electrical information is available from the network, signal processing can be used to estimate several parameters of the system such as impedance, power factors, power flow, stability, etc. where such information can be used by the system operator for more efficient control of the electric grid. The method presented in Fig. 1 can be named as passive monitoring or noninvasive monitoring, because the information is extracted from the system using noninvasive sensors allocated along it. In passive monitoring, no external perturbation is applied to the system. However, for some parameters, estimation by passive monitoring is not the most appropriate method, which can lead to inaccuracy. The classical example is for harmonic impedance estimation. The noninvasive methods rely on voltage and current harmonic variations caused by natural load variability [1-4]. The weakness of noninvasive methods rests in the fact that the harmonic content of the natural excitation cannot be

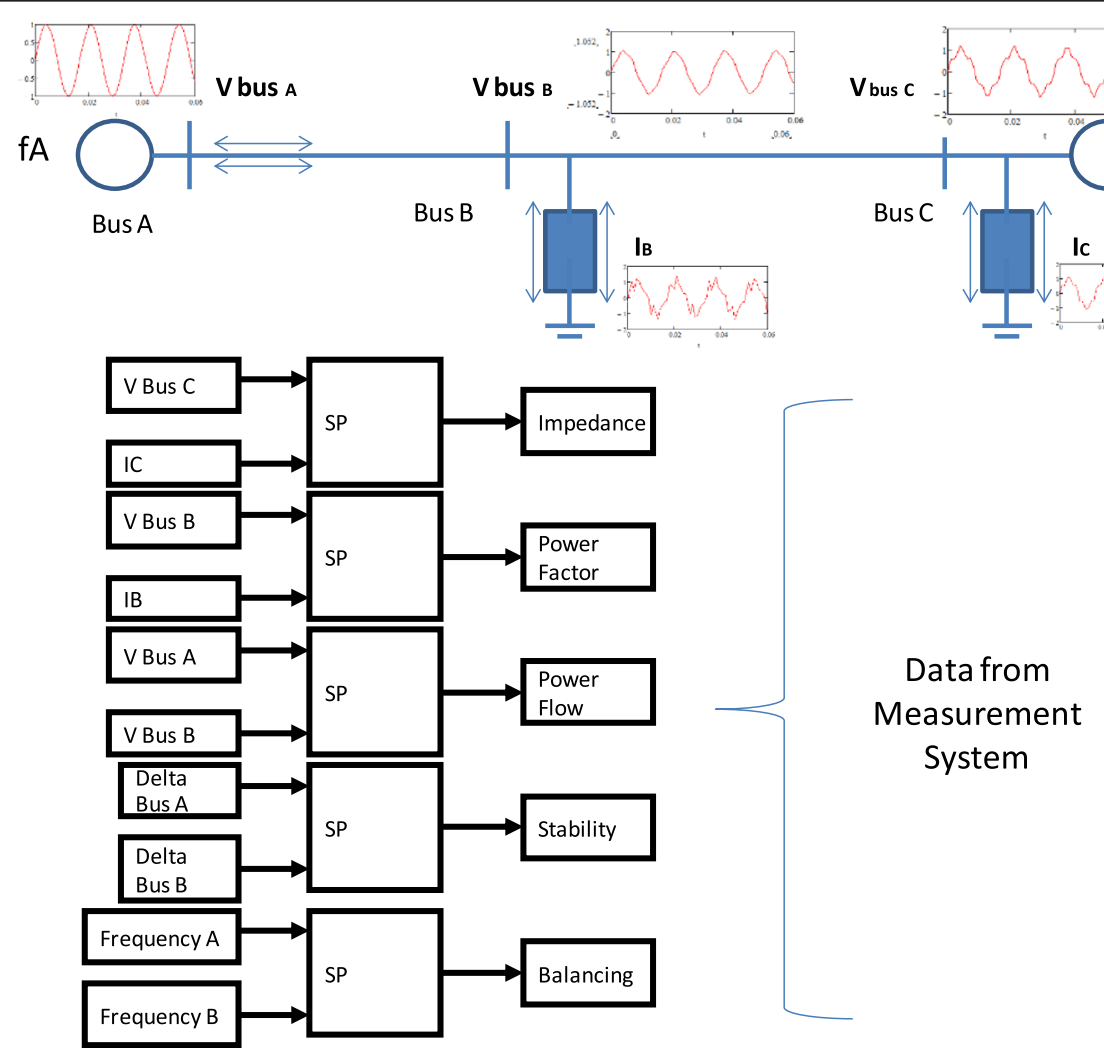

Fig. 1 A comprehensive system-wide signal processing analysis 
controlled; furthermore, the temporal natural excitation is sensitive to changes of background voltage harmonics as well as time variability of measured harmonic impedance itself. Thus, in order to achieve satisfactory accuracy, they require long-term recording and data averaging.

On the other hand, active invasive monitoring methods rely on the fact that an external disturbance is injected into the network and the resultant voltage/current is measured at the measuring point (MP) [5-12]. Such methods often have better accuracy than noninvasive methods but may cause interference in the electrical network, and the equipment used to inject the transient signal increases the cost in comparison to the noninvasive methods.

Invasive methods are generally divided into transientand steady-state measurement-based procedures. Transientbased methods use capacitor switching to provoke transient disturbances into the network to rate the frequencydependent network impedance by the ratio between the measured voltage and current. Otherwise, steady-statebased methods use voltage and current, pre- and postdisturbance, to determine network impedances for each harmonic frequency.

In [6], for example, the authors propose a steady-state method that injects a square-wave current waveform, with constant frequency and magnitude, in order to measure the harmonic impedance of a microgrid.

The method proposed in [7] uses a thyristor to create a controlled short circuit at the measurement point. The short circuit produces a pulse current and a voltage distortion, which is used to estimate the system impedance. The method is able to estimate the harmonic impedance even in the presence of nonlinear loads. The process uses the extraction of the transient signals from the recorded voltage and current waveforms by subtracting two consecutive cycles of the waveform. The transient signals are produced by using multiple firing angles to improve the precision at the frequencies with low transient current energy.

In [8], the method is based on processing the voltage and current transients generated when a power transformer is switched on. The discrete Fourier transform is applied to determine the harmonic components of voltage and current, and consequently, the harmonic impedance is calculated. However, this method is only suitable for the determination of low-order harmonic impedance values in AC supply systems.

In [9], the authors present an improved power system impedance measurement technique based on a power converter that injects a short-duration spike current at the MP. The advantage is that the width and weight of the spike can be controlled to minimize the disturbance on the grid. The fact that the spike length can be controlled means that the harmonic content injected may have some control; however, this control is not effective once the increasing of the frequency range requires the spike to be close to an impulse signal, which is not feasible.

Despite of the problems regarding the invasive method, such as costs, lack of frequency control, and network perturbation, they point to a very attractive methodology for identifying the electrical parameters and performing reliable diagnoses: the active monitoring and processing. Keeping the proportions in mind, active monitoring and processing are similar to medical examinations performed on patients, where a controlled signal or substance is injected to obtain accurate information about the health of the patients. The drawbacks of active monitoring can be minimized if part of the smart grid infra-structure is used to improve the system performance and reduce cost. This concept is supported by the fact that renewable generation will increase significantly in this new scenario. The power electronic interface for the renewable generation will be fundamental to the new generators, and it can be designed to inject power and exchange information within the network. In fact, the network parameter seen from the point where the generator will be installed is a relevant parameter for the voltage source inverter (VSI) control. For example, in [11, 12], current harmonic is injected from the VSI with the goal of grid impedance information.

Figure 2 shows a basic example of an active monitoring and processing system to impedance estimation. The electronic system to inject a transient signal can be standalone or be a part of a power electronic interface of a generator. The impedance of the system from the MP can be computed by injecting a transient signal and collecting the voltage and current at the MP. To be effective, the injected signal should have the following characteristics:

- Frequency controls, so the network can be excited at the frequency range of interest.

- The transient generated by the injected signal at network should be controlled, so the power system is minimally disturbed.

- The injected signal should be generated as simply as possible, which means at a low cost as possible.

A good waveform candidate to be injected is the Morlet wavelet (MW) or the modulated Gaussian signal (MGS) or any other signal that has finite support and has the three characteristics described before. The MGS was used in [13] for network impedance estimation ranges from the second harmonic up the 50th harmonic. Figure 3 shows an example of the MGS waveform for the 5th, 11th, and 30th harmonics, each one with time duration of half cycle of the fundamental component. These signals are injected sequentially including silence space of one cycle between two consecutive MGS signals. After the injection of each modulated signal, the SWRDFT [14] is used to process the signals and extract the impedance at 


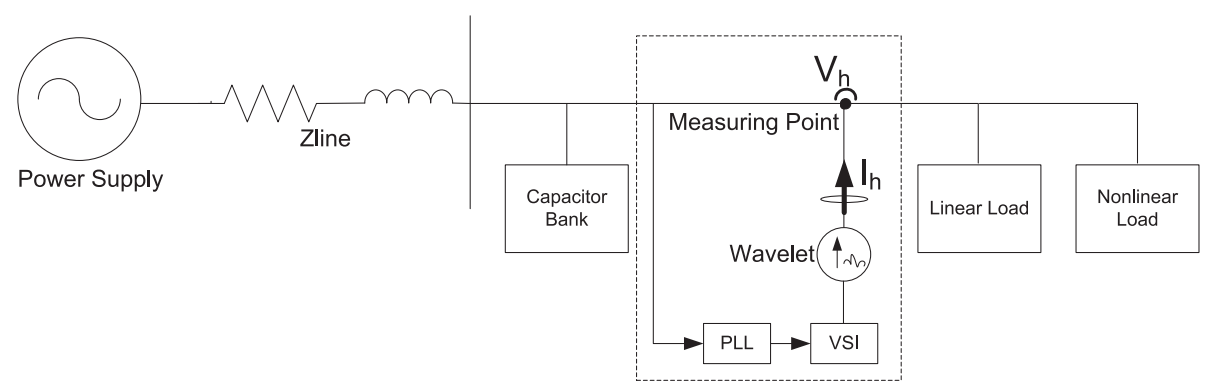

Fig. 2 Basic example of harmonic impedance measurement using active monitoring and processing

modulated frequency. The main advantage of this methodology is that instead of injecting a single signal that has energy in a large frequency range, small signals are injected in different frequencies, so it is possible to have control of all spectra of interest.

As an example to show the effectiveness of the proposed method, the modified IEEE 13-bus industrial distribution system [15] is used. The modification is due to the nonlinear loads (six-pulse AC/DC converters) included at buses 5, 10, 11, and 13 of Fig. 4. Figure 5 shows the magnitude and phase of the impedance. The results were simulated using the SimPowerSystems/Simulink. The estimated impedance was compared with the one obtained by the impedance meter block (IMB) of the Simulink. The difference between the ideal and estimated impedance values was lower than $3.85 \%$. The methodology of injecting a sequence of small signals of controlled frequencies seems to be promising.

\subsection{Where to go on active monitoring and processing}

The results presented above are a preamble of possibilities that can be explored through active monitoring and processing for diagnoses and electrical parameter estimation. In the new scenario of smart grid, the network complexity will require new methods to overcome new problems that are to come and some that are still a challenging topic. Active monitoring seems to be a useful tool to help with problems such as the harmonic distortion responsibility, islanding detection, resonance detection in the network, and smart power quality diagnostics.

\section{Sparse representation of power system signal}

Signal monitoring and data acquisition are increasingly being required in contemporary scenarios of power systems. Several applications need real-time monitoring and/ or offline analysis such as protection, control, and power quality (PQ) analysis. In many cases, acquisition and storage of raw data are required. This is sustained by the fact that the offline processing of this data can provide information not shown in the real-time analysis, allowing further system enhancement, troubleshooting, and algorithm optimization among others $[16,17]$. However, most of the conventional recorders are application oriented, acquiring either a short term of failure signal or a specific disturbance signal $[18,19]$.

The raw data recorded will always be of great importance in power systems and especially with the growth of smart grids. However, continuous raw data recording of electrical signal is not a simple task due the large amount of data to be recorded. In this way, an efficient disturbance detector [20] and data compressor algorithms assume important roles in the new smart grid scenario.

The discrete wavelet transform (DWT) [21, 22] has been extensively used in lossy compression applications.

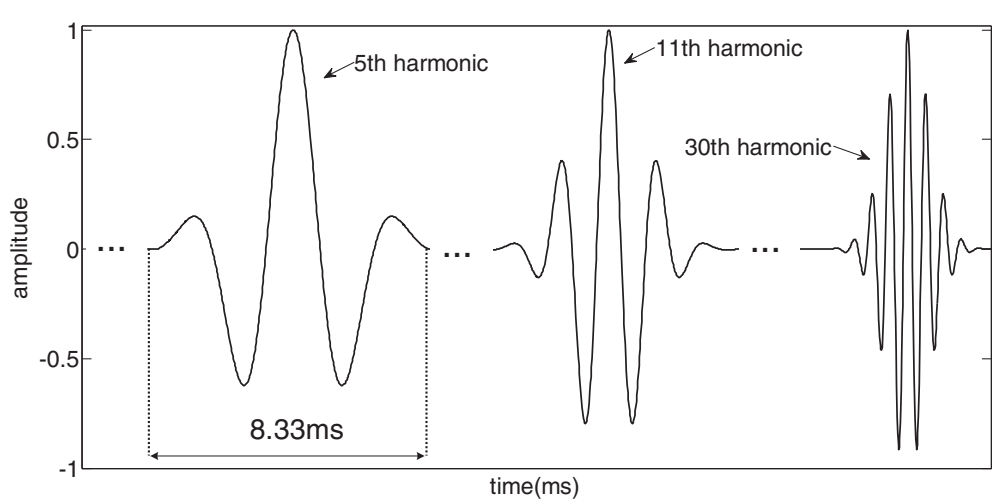

Fig. 3 MGC for different harmonic orders 


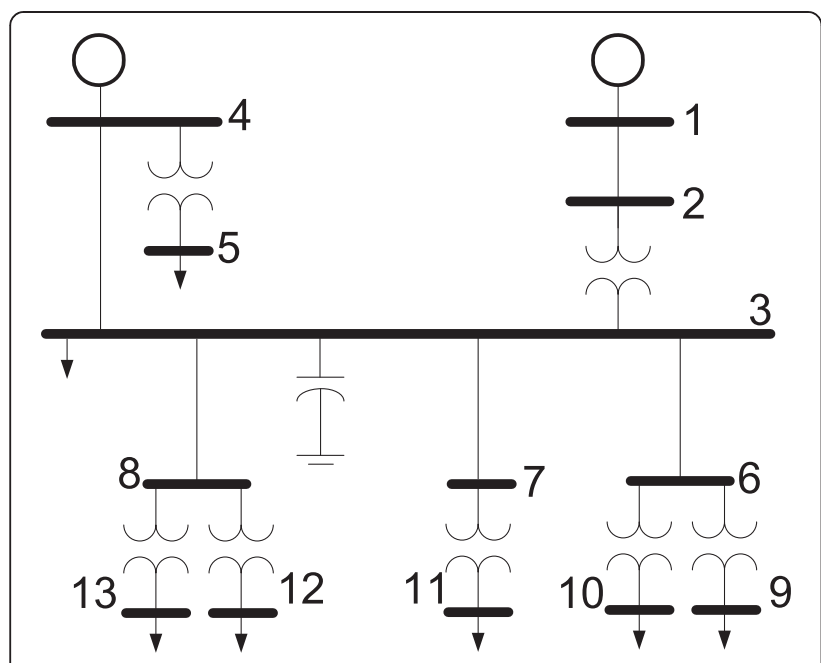

Fig. 4 A modified IEEE 13-bus industrial distribution system

However, recently, sparse representation [23-27] has emerged as a potential method for compression, as well as detection and estimation in power system applications. Thus, this section will present a brief review of this new signal processing technique and present promising results in compression application that can be part of a future PQ data or system performance recorder.

Despite the fact that most of the references on sparse representation focuses on image compression, there are some that address power system applications. In [24], the author uses sparsity constraints and adaptive pursuit techniques for transient detection in power system signals. In [25], the authors propose to carry out a coherent representation of electrical signals through adaptive decomposition using exponentially damped sinusoidal components, and for that, an intermediate step with Gabor atoms is necessary. In [26], the same authors eliminate the use of
Gabor atoms working directly with a dictionary formed by damped sinusoids.

The application of sparse approximation techniques faces two main problems: (i) algorithms for performing sparse decomposition and their performance analysis and (ii) dictionary composition methods. The most widely used algorithm for solving the first problem is the matching pursuit [27] or some of its variations. The second problem could be addressed in two ways: using a predefined dictionary or a learning strategy. The former is simpler and the latter more flexible. Since the compression application needs to be done online, the simpler option is the best choice.

If the dictionary represents a simple basis, the decomposition will be unique. However, comprehensive dictionaries are more useful since they allow different decompositions of the signal, and in this way, compression applications must seek the sparsest one. One strategy widely used is building the dictionary as a union of orthogonal basis. In power systems, the wavelet basis is widely used due to its capacity of representing transient phenomena; however, it is not efficient in representing harmonic components (a large number of wavelet coefficients are necessary). One basis that is efficient for harmonic representation is the Fourier basis. Thus, the idea of building a dictionary as a union of the wavelet and the Fourier bases seems to be very attractive. In this way, the efficiencies in transient and harmonic representations are put together.

In the following example, the dictionary was built containing sine and cosine up to the 50th harmonic order and wavelet signals derived from a Daubechies 3 and a tree of three levels. The size of the dictionary is 512 rows (number of samples of the signal) per 612 columns (100 harmonic and 512 wavelet components). The algorithm
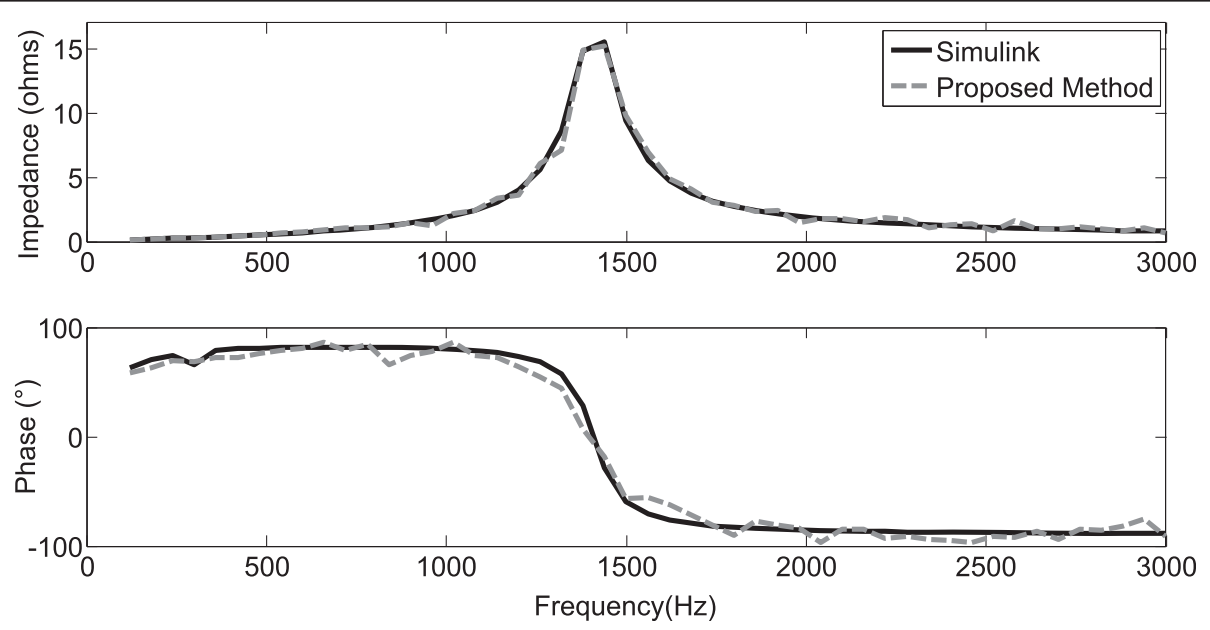

Fig. 5 Impedance for a modified 13-bus industrial distribution system, with linear and nonlinear loads 


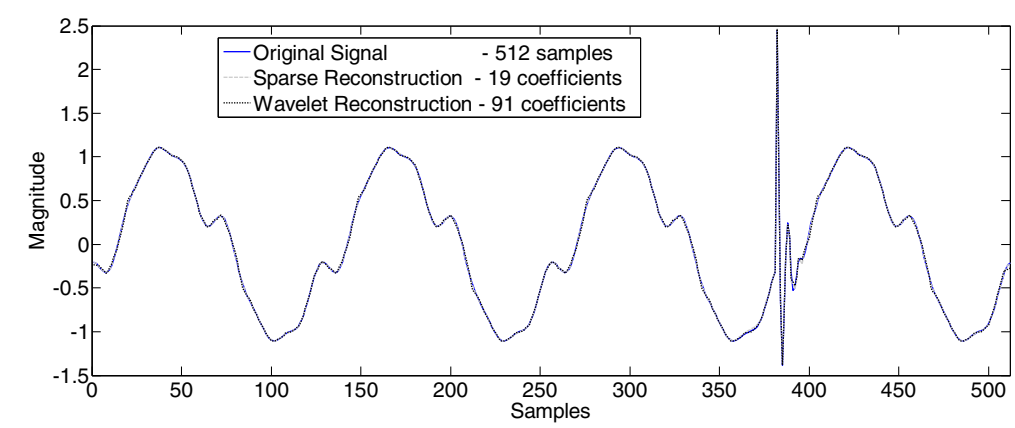

Fig. 6 Comparison of the compression methods: wavelet $\times$ sparse reconstruction

used to evaluate the sparse representation was the orthogonal matching pursuit (OMP) [27]. The OMP is a greedy algorithm that in each iteration searches for the dictionary element that has the biggest inner product with the residual.

The results of the OMP with the proposed dictionary were compared with a classical DWT using three levels of decomposition based on Daubechies 3 mother wavelet. Figure 6 shows a power signal containing 16, 10, and $7 \%$ of the third, fifth, and seventh harmonics respectively, besides the fundamental component and a transient at the beginning of the fourth cycle.

It can be noted in Fig. 6 that using the wavelet technique, 91 coefficients were necessary while using the sparse representation with the proposed dictionary, only 19 coefficients were used. As mentioned earlier, the composition of the proposed dictionary outperforms the DWT in the representation of stationary components.

Statistical tests were performed, and the results are shown in Table 1 when the two techniques were submitted to various disturbance signals, such as sag, swell, harmonics, and transients. The disturbance parameters were all randomly chosen for each generated signal.

Analyzing the results in Table 1 , it is clear that the sparse representation technique performs better than the wavelet. It is due to the proposed dictionary that uses a wavelet basis together with sinusoidal components, combining the capacity of representing transient phenomena of the wavelets with the ability of the Fourier basis in representing sinusoidal steady-state signals. One drawback of the sparse representation technique is

Table 1 Comparison of the methods [52]

\begin{tabular}{lll}
\hline Disturbance & DWT & Sparse \\
\hline Sag/swell & 69 & 31 \\
Harmonics & 103 & 13 \\
Transients & 87 & 25 \\
Harmonics + transients & 101 & 31 \\
\hline
\end{tabular}

the computational complexity that is related to the dictionary size. Therefore, dictionary structures must be studied in order to lower the dictionary dimension as well as the decomposition algorithms that are simpler and faster to run in real time.

\subsection{Where to go on sparse representation}

Sparse representation of signals has received a great attention in recent years. The search for a redundant dictionary that guarantees compact representation using atoms that has physical interpretation for the signal has two advantages: at the same time the signal is compressed efficiently, information regarding the signal composition can be obtained with direct help to classification and estimation task. A single example presented above, where the signal was composed of harmonic and transient, shows the ability of the method for the detection and classification tasks through the knowledge of the atoms used to represent the signal. Harmonic estimation follows directly by analyzing the energy of each Fourier atom added. The problem of sparse representation resides in the computation burden to reach the atom components and to find the best dictionary for the application. We believe that this new area has several possibilities for research and development of new solutions for smart grid.

\section{Real-time resampling technique}

Harmonic and interharmonic measurements are widely used in power quality (PQ) monitoring for characterizing the quality of energy and for troubleshooting. The FFT is the main signal processing tool used to characterize the magnitude spectrum of the input signal (voltage or current). However, that nonparametric tool, used for spectrum analysis, has well-known problems when the sampling rate is not synchronous with the fundamental component or when the signal is distorted by the interharmonic component [28-31]. Both can lead to misinterpretation of the spectrum content.

The asynchronous sampling rate leads to the spectral leakage that introduces spurious peaks into the computed 
spectrum that can be misunderstood as interharmonics. The spectrum leakage can be reduced if an appropriate window is used in the substitution of the rectangular window, or it can be eliminated by synchronization of the sampling rate.

The interharmonic causes the picket fence effects if the frequency falls between two adjacent bin components. The bin is the smallest resolution in the spectral analysis which is determined by the window length. The picket fence effect results in spectral leakage as well.

The International Electrotechnical Commission addresses the harmonic and interharmonic measurement in the IEC 61000-4-7 [32]. The standard defines the FFT as the digital signal processing tool applied to a data buffer obtained by synchronous sampling of the signal multiplied by a rectangular window. The buffer size must be such that it contains 10 integer cycles of the fundamental component for a $50-\mathrm{Hz}$ system or 12 integer cycles for a $60-\mathrm{Hz}$ system. This window length defines $5 \mathrm{~Hz}$ of frequency resolution. Furthermore, the maximum harmonic to be measured is defined to be the 50th harmonic.

A variety of methods have been developed in the area of harmonic and interharmonic analysis in the last decade. Some of them address the IEC standard and propose some kind of modification, such as the use of the Hanning window, Kalman filter, etc. [33-35]. However, the synchronization of the sampling rate is the main point to guarantee accuracy in the estimation of the harmonics and interharmonics.

The synchronization requires a frequency estimation algorithm. For this purpose, there are a lot of frequency estimation algorithms [36-41]. All of them work appropriately in a normal situation, which means in stationary or quasi-stationary signals; however, they are inaccurate in some situations such as the following: (i) the interharmonics (subharmonic) are close to the fundamental frequency and (ii) during sag or transient disturbs. In those cases, there are large deviations between the estimated frequency and the actual frequency, which compromise the accuracy of other parameters whose estimators are frequency dependent. While the IEC standard places a flag in the case of disturbance, warning the user that the processed information cannot be valid, there is no indication in the case of frequency deviation due the subharmonic presence. This deviation is seldom noticed in literature; however, with the growing of the renewable generation, the voltage fluctuation is expected to increase as well, and a new frequency estimation algorithm robust to voltage fluctuation must be investigated.

If the frequency estimation is available accurately, then synchronization can be performed by adapting the sampling time at the hardware level or by keeping the sampling time fixed and performing a resampling technique or time interpolation. The last approach is generally preferred by designers, since modification resides at the software level. Resampling or interpolation techniques can be performed using batch processing or sample by sample. In both cases, real-time processing and no-fractional resampling may be required. No-fractional resampling or interpolation means that the rate of interpolation is not an integer and in a more general case neither rational. For integer or fractional interpolation, there are good algorithm using an interpolator and decimator structure [42, 43]; however, as the system frequency varies continuously, the concept of arbitrary sampling rate interpolation (ASRI) should be used [44-51].

Figure 7 illustrates the original samples $x[n]$ and the new samples that need to be estimated, $y[n]$, using the new sampling time $T_{\mathrm{s}}^{\prime}$. The example shown in Fig. 7 illustrates the interpolated sample $y[m]$ requiring the original samples $x[n-1]$ to $x[n+3]$. Note that $m$ has been used as the index of the interpolated sequence $y[m]$ and $n$ as the index of the original sequence $x[n]$.

The ASRI of signal $x[n]$, originally sampled with a sampling frequency $F_{\mathrm{s}}$, consists of the following steps:

i) Estimation of the fundamental frequency $f_{1}$ from the samples of the input signal $x[n]$

ii) Calculation of the resampling moments $\alpha$

iii) Resampling of the signal $x[n]$ at the moments $2+\alpha$ using interpolation in time domain, giving rise to the output signal $y[n]$

Figure 8 shows a block diagram for dynamic resampling. After parameter initialization, the actual system frequency is estimated and the new sampling frequency is obtained; then, the relationship between the new sampling frequency and the ideal sampling frequency (60 or $50 \mathrm{~Hz}$ ) is calculated, generating the factor $\lambda$. From this factor, the $\alpha$ is found and then the interpolation of the new sample is carried out. Figure 8 shows a practical implementation of the ASRI. This structure is known as the Farrow structure [42], and the filters $H_{i}(z), i=0,1$, 2 , and 3 , depend on the type of interpolation function used, for example, for the third Lagrange polynomial, the filters are $[51,52]$

$$
\begin{gathered}
H_{0}(z)=-\frac{1}{6} z^{-2}+\frac{1}{2} z^{-1}-\frac{1}{2}+\frac{1}{6} z \\
H_{1}(z)=\frac{1}{2} z^{-1}-1+\frac{1}{2} z \\
H_{2}(z)=-\frac{1}{6} z^{-2}-z^{-1}+\frac{1}{2}+\frac{1}{3} z
\end{gathered}
$$

and for the third-order spline function, the filters are 


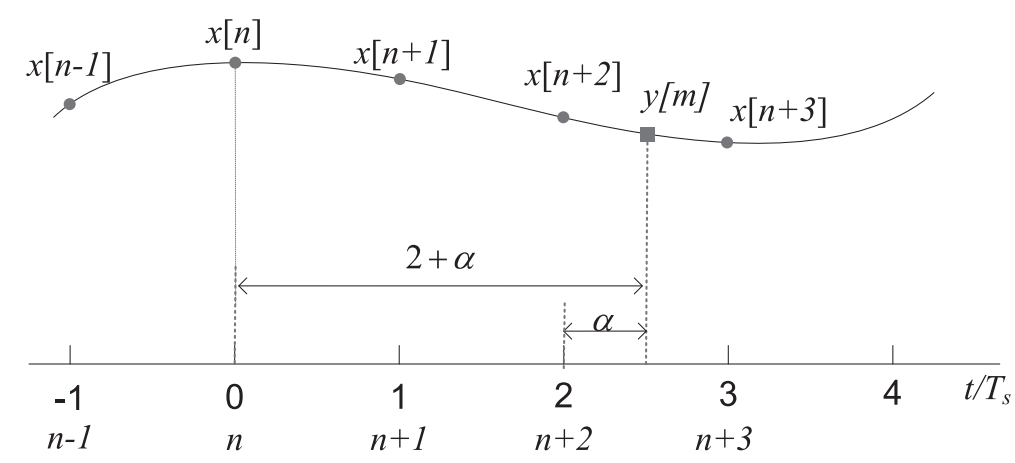

Fig. 7 Illustration of real-time resampling processing

$$
\begin{gathered}
H_{0}(z)=-\frac{1}{6} z^{-1}+\frac{1}{2}-\frac{1}{2} z+\frac{1}{6} z^{2} \\
H_{1}(z)=\frac{1}{2} z^{-1}-1+\frac{1}{2} z \\
H_{2}(z)=-\frac{1}{2} z^{-1}+\frac{1}{2} z \\
H_{3}(z)=\frac{1}{6} z^{-1}+\frac{2}{3}+\frac{1}{6} z
\end{gathered}
$$

Figure 9 shows a feedback branch controlled by the value of $\alpha$. While the value of $\alpha \leq 1$, the interpolated output is computed without change in the filter's memory. The value of $\alpha$ is modified in each step that a new output is generated. If $\alpha>1$, the $\alpha$ value is modified as presented in Fig. 9 and the filter's memories are updated. The algorithm continues indefinitely.

The main points to be considered in choosing the interpolation function are as follows:

- Spline interpolation does not pass through the knot points; this means that some small errors are

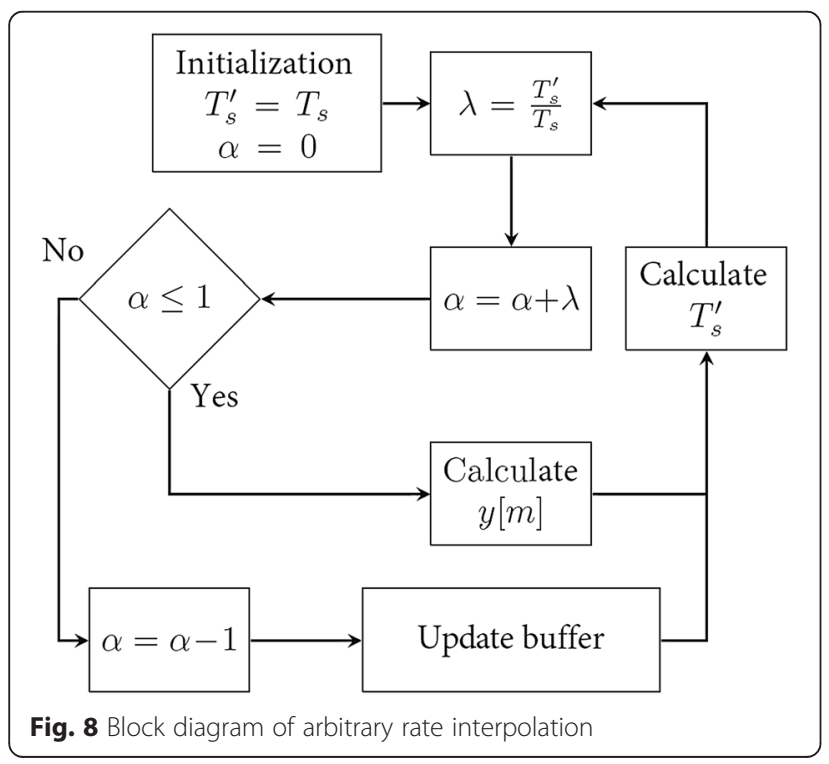

expected at the knot points. To reduce these errors, the B-spline least square [47] and the B-spline inverse function $[53,54]$ are used.

- If the sampling frequency is much higher than the maximum frequency present in the signal that will be interpolated. The Lagrange interpolator leads to better results with smaller computational effort; otherwise, the spline interpolator should be preferred.

In order to compare the errors produced by ASRI, consider the case where the fundamental frequency is timevarying frequency, varying from 60 to $61 \mathrm{~Hz}$ in $1 \mathrm{~s}$ (ramp variation). Figure 10 compares the error in the harmonic groups of the IEC 61000-4-7 [55], without interpolation, after interpolation using a third-order B-spline (B-spline), third-order Lagrange polynomial (Lagrange), and using offline B-spline interpolation (offline interpolation). In this last interpolation method, the average frequency of the window was taken as constant. The harmonic component orders present at the signal were $h=[3,7,10,11,13,15$, $19-21,27,31,37,45,50]$, and the amplitude of the harmonics was all $0.2 \mathrm{pu}$. The sampling frequency was $128 * 60 \mathrm{~Hz}$ and a signal to noise rate (SNR) of $40 \mathrm{~dB}$ was added to the signal. As can be observed from these results, the B-spline LS presented an error lower than $0.5 \%$ for all groups, while the Lagrange reaches an error higher than $3 \%$ and the offline B-spline considering the average frequency reaches about $12 \%$. The worst case is when noninterpolation is considered; in this situation, error approaches $90 \%$. These results show that ASRI produces better results than considering the fundamental frequency fixed inside the window.

\subsection{Where to go on real-time resampling}

Multirate systems require different sampling rates for adequate signal processing. As a consequence, sampling alteration becomes a necessity as the different techniques are used to analyze the same signal. This section discussed the arbitrary sampling alteration, a very important point when the frequency varies continuously. As the electrical 


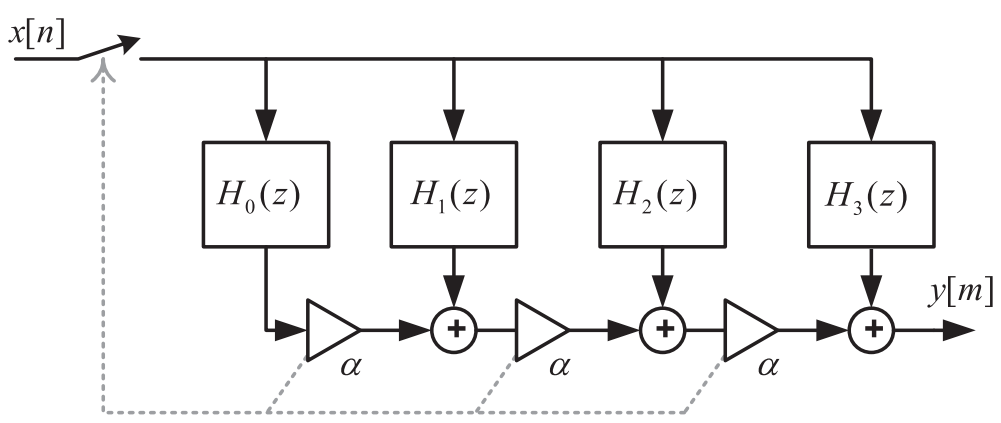

Fig. 9 Implementation of the arbitrary rate interpolator using the Farrow structure

signal of the future smart grid becomes highly time varying and a variety of analyses will have to be done on the same signal, the adequate sampling rate for the different parameters being extracted becomes an important aspect of the overall signal processing analysis. Again, a good frequency estimator is the key for the successes of the interpolation techniques.

\section{Time-frequency applied to power fluctuations caused by intermittent renewable generation and load variations}

A time-frequency method, such as the wavelet transform (WT), is an appropriate tool for the analysis of nonstationary signals. Unlike conventional frequency analysis methods, wavelets give information about the range of frequency components of a signal as a function of time. The first time that WT was suggested to be applied in the power system area was in the IEEE Working Group on Harmonic Modeling Simulation in 1993 and subsequently published in 1994 [56]. After that, several works have been published addressing power system application with emphasis on the following areas: power quality analysis and monitoring, power system protection [57], wind power forecasting [58, 59], and, more recently, fluctuation identification caused by renewable energy sources (RES) [52, 60]. This section explores the application of WT in generation and load profiles. Through the analysis of relevant signals, the variations in the signals of different frequency ranges can be characterized. In this section, the power fluctuations in a small $10-\mathrm{kV}$ distribution network (acting as a microgrid) are analyzed using WT.

For a signal $P(t)$, the wavelet representation can be written as [52]

$$
P(t)=\sum_{k} \sum_{n} P_{\mathrm{DWT}}(k, n) \psi_{k, n}(t)
$$

where the factors $P_{\mathrm{DWT}}(k, n)$ are the wavelet coefficients that indicate the presence of transformed wavelet components at different scaling factors in the original signal at different time shifts. For all signals here mentioned,

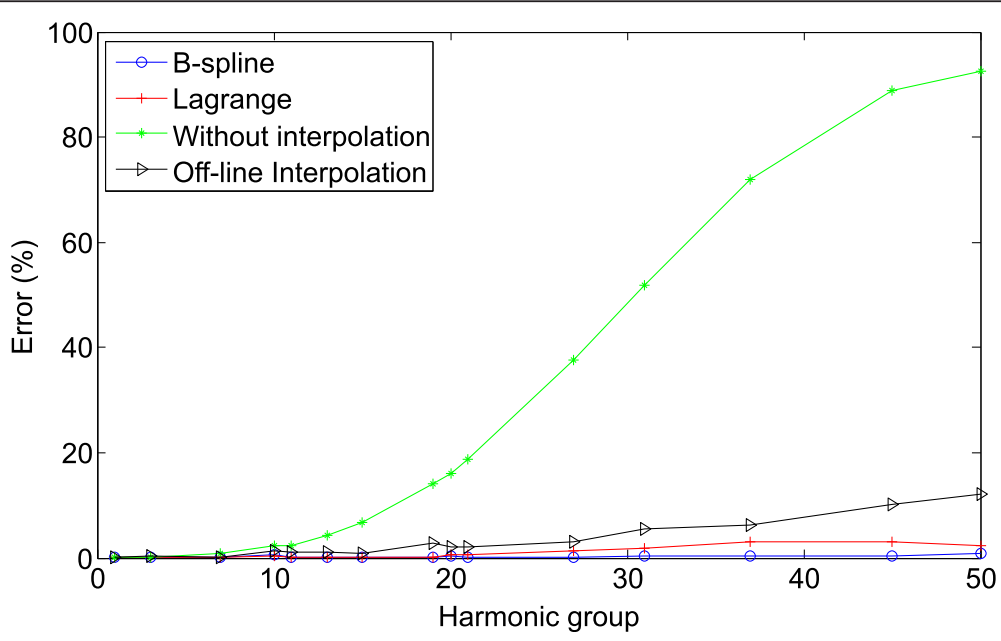

Fig. 10 Errors in the harmonic group estimation after interpolation 
the signal is normalized using Eq. 4 to be within $[0,1]$ and the Meyer wavelet is used as the mother wavelet.

$$
P_{\text {norm }}(t)=\frac{P(t)-\min [P(t)]}{\max [P(t)]-\min [P(t)]}
$$

The maximum scaling factor $L$ for the wavelet decomposition follows from the lowest frequency to be analyzed and its sampling frequency $F_{\mathrm{s}}$. These can be found in Eq. 5:

$$
F_{k}=\left\{\begin{array}{l}
\left(\frac{F_{\mathrm{s}}}{2^{k+1}}, \frac{F_{\mathrm{s}}}{2^{k}}\right) \\
\left(0, \frac{F_{\mathrm{s}}}{2^{k+1}}\right), k=L
\end{array}, k=1,2, \ldots, L-1\right.
$$

where $F_{k}$ indicates the frequency band related with the wavelet coefficient in the scale, or level, $k$.

From all wavelet components $P_{\mathrm{DWT}}(k, n)$ that represent a certain frequency range, a number of components are selected that contribute most to the original signal $P(t)$ as follows from Eq. 3. The selection of these components is based on the root mean square (RMS) value of each individual component.

The components considered to contribute most are the ones with the highest RMS values, as derived with Eq. 6 . As such, the most relevant scaling factors of $P_{\mathrm{DWT}}(k, n)$ can be used to approximate the original signal. This is based on the superposition of the most relevant scaling factors as, indicated in Eq. 3. These relevant scaling factors can be investigated as well, as each component holds the information of the original signal within a certain frequency bandwidth; the signal reconstruct in this way is named here as synthetic signal. When the individual relevant scaling factors are determined, they reveal additional information about fluctuations in the original signal at specific time periods. From now on, the wavelet coefficients $P_{\mathrm{DWT}}(k, n)$ will be renamed $A_{k, n}$, for the sake of simplification, then

$$
A_{k, \mathrm{RMS}}=\sqrt{\frac{1}{N+1} \sum_{n=0}^{N} A_{k, n}^{2}}
$$

where $N$ is the number of samples in each scale.

\subsection{A smart microgrid example}

In order to illustrate the use of wavelet for identification of power fluctuations, a small grid is used as an example. The microgrid in this study consists of a number of loads connected to a small $10-\mathrm{kV}$ radial distribution network containing a 2-MW wind turbine (W) and a conventional generator $(G)$. The microgrid is to be operated in island mode, and therefore, the conventional generator needs to be able to deal with the aggregated fluctuations of load and wind turbine. The network topology is illustrated in Fig. 11.

The power $P_{\mathrm{G}}(t)$ that the conventional generator $\mathrm{G}$ needs to produce is given by Eq. 7:

$$
P_{\mathrm{G}}(t)=\sum P_{\text {load }}(t)+\sum P_{\text {losses }}(t)-P_{\text {wind }}(t)
$$

where $P_{\text {load }}(t)$ is the power of each load, $P_{\text {losses }}(t)$ are the network losses, and $P_{\text {wind }}(t)$ is the power generated by the wind turbine.

After completing the load flow simulations and normalizing the power profile using Eq. 4, the power to be generated by generator $G$ was investigated using the wavelet methodology to determine its characteristic fluctuations to be managed by generator G. The load flow simulations were done for each minute during a month, so the sampling period $\left(1 / F_{\mathrm{s}}\right)$ of $P_{\mathrm{G}}(t)$ is $1 \mathrm{~min}$. Using Eq. 5, the maximum scaling factor was decided to be 14 . The three components of $A_{k, n}$ with the highest RMS values, obtained from Eq. 6, were chosen to be the most relevant. They were the components $A_{10, n}, A_{11, n}$, and $A_{12, n}$. Note, in Fig. 12, that the reconstructed (synthetic) signal using only these three components is close to the actual signal.

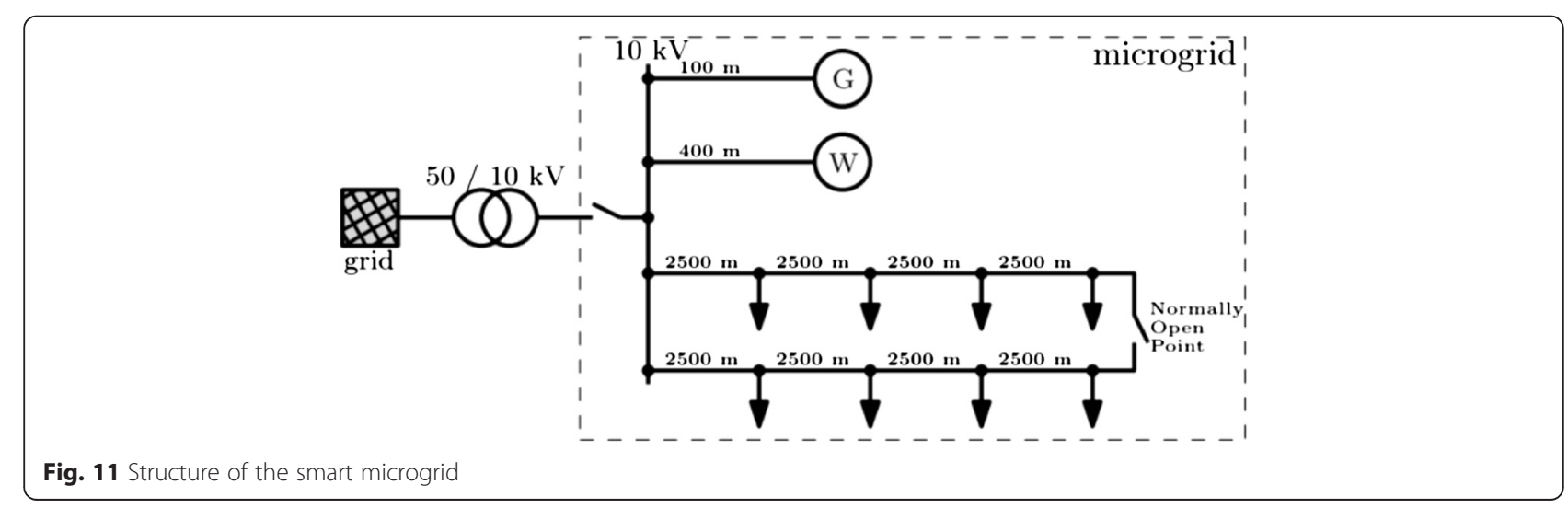




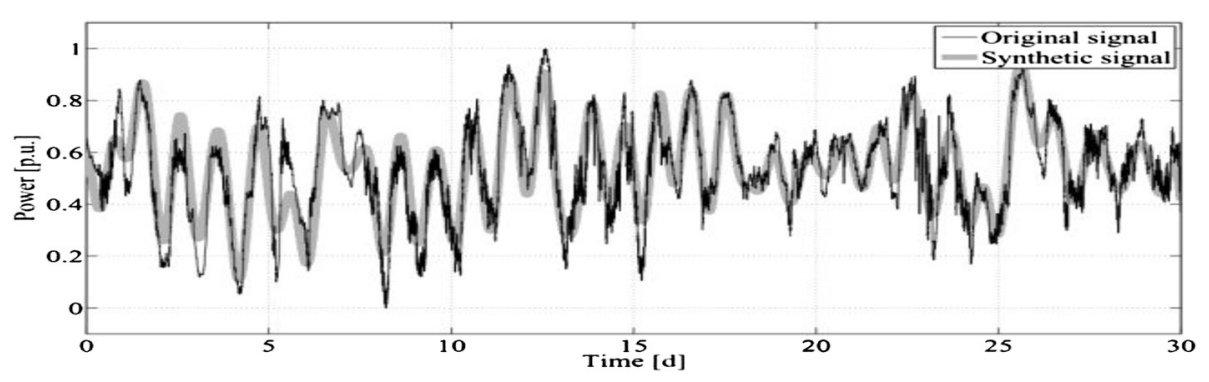

Fig. 12 Original and synthesized power profiles of generator G during 1 month

The power profile for generator $\mathrm{G}$ can be summarized from Fig. 13. In this figure, the components $A_{10, n}, A_{11, n}$, and $A_{12, n}$ together with the moving average component, $A_{M, n}$, are shown. Note that $A_{10, n}$ presents higher frequency and significant energy, so the generator must follow this profile. The period of this component can be found as follows.

The time sampling $T_{s, k}$ of the center of each level $k$ can be determined from Eq. 5 as

$$
T_{s, k}=\left(2^{k+1} / F_{\mathrm{s}}+2^{k} / F_{\mathrm{s}}\right) / 2=2^{k}\left(1,5 / F_{\mathrm{s}}\right)
$$

For the present example, $1 / F_{\mathrm{s}}=60 \mathrm{~s}$, so

$$
T_{s, k}=90 \times 2^{k} \mathbf{s}
$$

From Fig. 13, it can be concluded that $A_{10, n}$ is the component with a daily profile $\left(T_{s, k}=2^{10} .90 \mathrm{~s} \Rightarrow\right.$ 1.07 days) and has the largest share in the original signal. Generator $\mathrm{G}$ must therefore be able to ramp up and down within this period. If generator $\mathrm{G}$ is able to follow the fluctuations within a 1-day period, it will be able to produce the synthetic profile as shown in Fig. 13. To provide the difference in power between the synthesized and the original profiles, an electricity storage device can be added to the microgrid under study. As shown in this example, the wavelet analysis can be used to characterize both the generator $\mathrm{G}$ and the required electricity storage device.

\subsection{Where to go on wavelet application on smart grid}

The increasing complexity of the electric grid requires new signal processing techniques which can be used to analyze power systems. It is proposed, for example, that wavelet analysis can be applied to determine fluctuation patterns in generation and load profiles. This is achieved by the filtering of its wavelet components based on their RMS values, and it is possible to identify the most relevant scaling factors from the analysis.

The application of wavelet analysis, as described in this section, may prove useful both for the characterization of possible electricity storage devices and the determination of the required balancing capacities. It can also aid energy companies to make improved bids in energy markets by having specific information on the characteristic fluctuations of its renewable generation, and it provides them with the ability to counteract these by using conventional generation and electricity storage. In the future, the application of wavelets to analyze generation and load signals may prove very useful for agents responsible for the operation and control of the network.

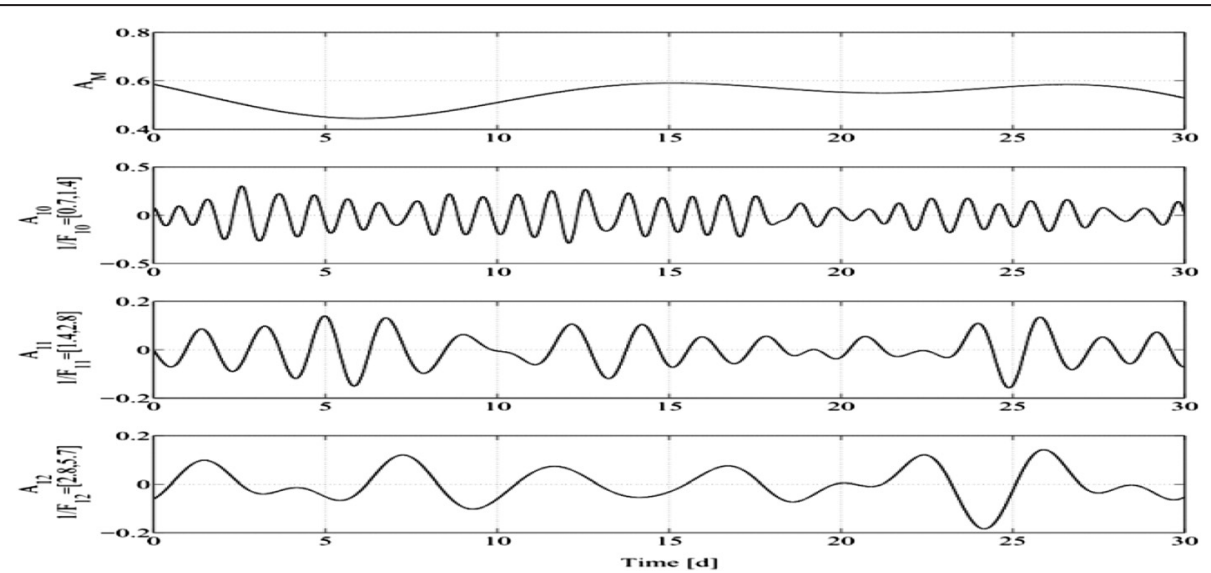

Fig. 13 Most relevant wavelet components for the microgrid simulation during 1 month and the moving average component [52] 
These agents could use wavelet analysis to improve their response performance. Moreover, wavelet analysis can be applied to analyze price signals in these time-dependent energy markets.

\section{Conclusions}

This paper presented some advantageous application of signal processing in the context of smart grid. With the growing complexity of power system network characterized by the proliferation of disperse, distributed, and renewable generation and the introduction of new nonlinear generation and loads, some common tasks tend to be so multifaceted that traditional techniques will not be able to deal with them appropriately. Advanced signal processing techniques will be essential to support the tasks of protection, operation, monitoring, and expansion, among others. Thus, one of the skills that a new professional in the power system field must have is the ability to use interchangeably a number of advanced signal processing techniques already available.

\section{Competing interests}

The authors declare that they have no competing interests.

\section{Acknowledgements}

The authors would like to thank CAPES, CNPq, and FAPEMIG for supporting this work.

\section{Author details \\ ${ }^{1}$ Federal University of Juiz de Fora, Juiz de Fora, MG, Brazil. ${ }^{2}$ Federal University of Itajubá, Itajubá, MG, Brazil.}

Received: 27 November 2014 Accepted: 30 April 2015

Published online: 15 May 2015

\section{References}

1. W Xu, EE Ahmed, X Zhang, X Liu, Measurement of network harmonic impedances: practical implementation issues and their solutions. IEEE Trans. Power Delivery 17(1), 210-216 (2002)

2. H Jin, W Freitas, JCM Vieira, Y Honggeng, L Yamei, Utility harmonic impedance measurement based on data selection. IEEE Trans. Power Delivery 27(no. 4), 2193-2202 (2012)

3. Y Xu, S Huang, Y Liu, Partial least-squares regression based harmonic emission level assessing at the point of common coupling. Presented at the Int. Conf. Power Syst. Technol., Chongqing, China, Oct. 2006.

4. J Hui, $\mathrm{H}$ Yang, $\mathrm{S}$ Lin, M Ye, Assessing utility harmonic impedance based on the covariance characteristic of random vectors. IEEE Trans. Power Delivery 25(3), 1778-1786 (2010)

5. W Wiechowski, J Lykkegaard, Bak-Jensen, CL Bak, Validation techniques of network harmonic models based on switching of a series linear component and measuring resultant harmonic increments. 9th International Conference on Electrical Power Quality and Utilisation, EPQU, 2007, pp. 1,6, 9-11 Oct. 2007

6. L Hou, H Shi, Z Yang, F Zhuo, Harmonic impedance calculation and measurement for an islanded microgrid. ECCE Asia Downunder (ECCE Asia), 2013 IEEE, pp. 550-554, 3-6 June 2013

7. W Wang, EE Nino, W Xu, Harmonic impedance measurement using a thyristor-controlled short circuit. IET Gener. Transm. Distrib. 1(5), 707-713 (2007)

8. C Xie, SB Tennakoon, R Langella, D Gallo, A Testa, A Wixon, Harmonic impedance measurement of $25 \mathrm{kV}$ single phase AC supply systems. Ninth International Conference on Harmonics and Quality of Power, 2000, vol. 1, pp. 214-219

9. M Sumner, B Palethorpe, DWP Thomas, Impedance measurement for improved power quality — part I: the measurement technique. IEEE Trans. Power Delivery 19(3), 1442-1448 (2004)
10. $Y$ Zhen, S Hongtao, Y Xiaolong, $\mathrm{H}$ Lixiang, Z Fang, Harmonic impedance measurement for an islanded microgrid using current injection (2012 IEEE 7th International Power Electronics and Motion Control Conference - ECCE Asia, Harbin, China, 2012)

11. L Asiminoaei, R Teodorescu, F Blaabjerg, U Borup, Implementation and test of an online embedded grid impedance estimation technique for PV inverters. IEEE Trans. On Industrial Electron. 52(no. 4) (2005)

12. M Ciobotaru, R Teodorescu, F Blaabjerg, On-line grid impedance estimation based on harmonic injection for grid-connected PV inverter. IEEE International Symposium on Industrial Electronics, 2007. ISIE, 2007

13. MO Prates, CA Duque, PG Barbosa, AS Cerqueira, A Testa, PF Ribeiro, Power system impedance measurement based on wavelet voltage imposed. 16th International Conference on Harmonics and Quality of Power (ICHQP), 2014, pp. 798, 802, 25-28 May 2014.

14. PM Silveira, C Duque, T Baldwin, PF Ribeiro, Sliding window recursive DFT with dyadic downsampling - a new strategy for time-varying power harmonic decomposition. Power \& Energy Society General Meeting, 2009: PES '09. IEEE, pp. 1-6, 26-30 July 2009

15. IEEE Standard 399-1990, IEEE recommended practice for industrial and commercial power system analysis (IEEE, New York, 1990)

16. H Maass, HK Cakmak, W Suess, A Quinte, W Jakob, K-U Stucky, U G Kuehnapfel, First evaluation results using the new electrical data recorder for power grid analysis. IEEE Trans. Instrum. Meas. 62, 2384-2390 (2013)

17. R Zivanovic, P Schegner, O Seifert, G Pilz, Identification of the resonantgrounded system parameters by evaluating fault measurement records. IEEE Transactions on Power Delivery 19(no. 3), 1085-1090 (2004)

18. M Moreto, JG Rolim, Using phasor data records and sequence of events to automate the classification of disturbances of power generating units. Electr. Power Syst. Res. 81(7), 1266-1273 (2011)

19. C Qiong, W Zhao-Hui, Research and design of portable fault recorder based on FPGA. Energy Procedia 12, 686-692 (2011)

20. CA Duque, MV Ribeiro, FR Ramos, I Szczupak, Power quality event detection based on the divide and conquer principle and innovation concept. IEEE Trans. Power Delivery 20(4), 2361-2369 (2005)

21. M Zhang, K Li, Y Hu, A high efficient compression method for power quality applications. IEEE Trans. Instrum. Meas. 60(6), 1976-1985 (2011)

22. F Bezerra, Costa, Fault-induced transient detection based on real-time analysis of the wavelet coefficient energy. IEEE Trans. Power Delivery 29(1), 140-153 (2014)

23. M Elad, Sparse and redundant representations: from theory to applications in signal and image processing. (Springer, 2010)

24. J Cooper, Sparse representations in power systems signals, 2009

25. L Lovisolo, et al. Efficient coherent adaptive representations of monitored electric signals in power systems using damped sinusoids. IEEE Trans. Signal Process. (2005)

26. L Lovisolo, et al. Modeling of electric disturbance signals using damped sinusoids via atomic decompositions and its applications. EURASIP Journal on Applied Signal Processing, Hindawi Publishing Corp., vol. 2007, n. 1, p. 168, 2007

27. S Mallat, Z Zhang, Matching pursuits with time-frequency dictionaries. IEEE Trans. Signal Process. (1993)

28. A Testa, MF Akram, R Burch, G Carpinelli, G Chang, V Dinavahi, C Hatziadoniu, WM Grady, E Gunther, M Halpin, P Lehn, Y Liu, R Langella, M Lowenstein, A Medina, T Ortmeyer, S Ranade, P Ribeiro, N Watson, J Wikston, W Xu, Interharmonics: theory and modeling. IEEE Trans. Power Delivery 22(4), 2335-2348 (2007)

29. R Yacamini, Power system harmonics. IV. Interharmonics. Power Eng. J. 10(4), 185-193 (1996)

30. C Li, W Xu, T Tayjasanant, Interharmonics: basic concepts and techniques for their detection and measurement. Electr. Power Syst. Res. 66, 39-48 (2003)

31. Z Liu, J Himmel, KW Bonfig, Improved processing of harmonics and interharmonics by time-domain averaging. IEEE Trans. Power Delivery 20(4), 2370-2380 (2005)

32. International Electrotechnical Commission: IEC 61000-4-7, Testing and measurement techniques - general guide on harmonics and interharmonics measurements and instrumentation, for power supply systems and equipment connected thereto, 2002

33. GW Chang, Cheng-I Chen, Measurement techniques for stationary and time-varying harmonics. Power and Energy Society General Meeting, 2010 IEEE, pp. 1-5, 25-29 July 2010

34. J Valenzuela, J Pontt, Real-time interharmonics detection and measurement based on FFT algorithm. Appl. Electronics, pp. 259-264, 9-10 Sept 2009. 
35. D Gallo, R Langella, A Testa, On the processing of harmonics and interharmonics in electrical power systems. IEEE Power Engineering Society Winter Meeting, vol. 3, pp. 1581-1586, 23-27 Jan 2000

36. TS Sidhu, Accurate measurement of power system frequency using a digital signal processing technique. IEEE Trans. Instrum. Meas. 48(№. 1), 75-81 (1999)

37. MD Kusljevic, JJ Tomic, LD Jovanovic, Frequency estimation of three-phase power system using weighted-least-square algorithm and adaptive FIR filtering. IEEE Trans. Instrum. Meas. 59(2), 322-329 (2010)

38. J Zhou, G Li, Plain gradient based direct frequency estimation using second-order constrained adaptive IIR notch filter. Electr. Lett. pp. 351-352 (2004)

39. $T(\mathrm{Li}, \mathrm{J}$ Jiang, Novel adaptive IIR filter for frequency estimation and tracking [DSP Tips\&Tricks]. 2009, pp. 186-189.

40. AK Ziarani, A Konrad, A method of extraction of nonstationary sinusoids. Signal Process. 84(n. 8), 1323-1346 (2004)

41. A Routray, AK Pradhan, KP Rao, A novel Kalman filter for frequency estimation of distorted signals in power systems. IEEE Trans. Instrum. Meas. 51(3), 469-479 (2002)

42. SK Mitra, Digital signal processing: a computer approach. s.l.: Mc Graw Hill, 2006.

43. PP Vaidyanathan, Multirate systems and filter banks ((Prentice Hall, Englewood Cliffs, New Jersey, 1993)

44. SR Dooley, RW Stewart, TS Durrani, Fast on-line B-spline interpolation. Electron. Lett. 35, 1130-1131 (1999)

45. G Evangelista. Design of digital systems for arbitrary sampling rate conversion. EURASIP Signal Process. 83, no. 2377-387

46. TA Ramstad, Digital methods for conversion between arbitrary sampling frequencies. IEEE Trans. Acoust. Speech. Signal Process. ASSP-32(NO. 3), 577-591 (1984)

47. D Borkowski, B Andrzej, Improvement of accuracy of power system spectral analysis by coherent resampling. IEEE Trans. Power Delivery 24(NO. 3), 1004-1013 (2009)

48. MA Lima, et al., A method of dynamic resampling for DFT-based harmonic analysis under time-varying frequency conditions. 14th International Conference on Harmonics and Quality of Power (ICHQP), 2010, pp. 1-6

49. U Zolzer, Digital audio signal processing (Wiley, New York NY, 1997)

50. S Cucchi, F Desinan, G Sicuranza, DSP implementation of arbitrary sampling frequency conversion for high quality sound application (Proc. IEEE International Conference on Acoustics, Speech, and Signal Processing, Toronto Canada, 1991), pp. 3609-3612

51. SK Mitra, Digital signal processing: a computer approach. s.l.: Mc Graw Hill, 2006

52. PF Ribeiro, CA Duque, PM Silveira, AS Cerqueira, Power systems signal processing for smart grids. (John Wiley \& Sons, 2013)

53. D Petrinović, Causal cubic splines: formulations, interpolation properties and implementations. IEEE Trans. Signal Process. 56(11), 5442-5453 (2008)

54. M Unser, Splines: a perfect fit for signal and image processing. IEEE Signal Process. Mag. 16(6), 22-38 (1999)

55. Standard, I. E. C. "61000-4-7." General guide on harmonics and interharmonics measurements for power supply systems and equipment connected thereto (2002).

56. PF Ribeiro, Wavelet transform: an advanced tool for analyzing non-stationary distortions in power systems, ICHPS VI/94 Italy

57. AW Galli, GT Heydt, PF Ribeiro, Exploring the power of wavelet analysis. IEEE Comput. Applicat. Power 9, 37-41 (1996)

58. L Dong, L Wang, X Liao, Y Gao, Y Li, Z Wang, Prediction of wind power generation based on time series wavelet transform for large wind farm. In Proc. 3rd Int. Conf. Power Electron. Syst. and Applicat., (Hong Kong, 2009), pp. 1-4.

59. C Lei, L Ran, Short-term wind speed forecasting model for wind farm based on wavelet decomposition. In Proc. 3rd Int. Conf. Electric Utility Deregulation and Restructuring and Power Technologies, (Nanjuing, China, 2008), pp. 2525-2529.

60. CH Lee, YJ Wang, WL Huang, A literature survey of wavelets in power engineering applications. Proc. Natl. Sci. Counc. ROC (A) 24, 249-258 (2000)

\section{Submit your manuscript to a SpringerOpen ${ }^{\circ}$ journal and benefit from:}

- Convenient online submission

- Rigorous peer review

- Immediate publication on acceptance

- Open access: articles freely available online

- High visibility within the field

- Retaining the copyright to your article

Submit your next manuscript at $\gg$ springeropen.com 